\title{
Application Research of Atmospheric Pressure Plasma on Microbial Breeding Physical Mutagenesis Technology
}

\author{
Jialin Guo \\ Luohe Medical College, Luohe, Henan, 462000
}

Keywords: microbial breeding; physical mutagenesis, ARTP; spore-forming bacteria

\begin{abstract}
Microbial physical mutation breeding technology is widely used to improve the characteristics of microbial strains, increase the yield and quality of microbial products, and also has important application value in biofuels and bioremediation. This paper focuses on the application of atmospheric pressure room temperature plasma (ARTP) physical mutagenesis technology in microbial mutation breeding, which has obvious mutagenic superiority to spore-forming species with important industrial and agricultural application value. The future development trend and important application prospects of microbial physical mutation breeding technology are further analyzed, which provides reference for microbial physical mutation breeding.
\end{abstract}

\section{Introduction}

With the development of society, the application range of microorganisms is more and more extensive. Microbial products are inseparable in all aspects of pharmaceutical production, environmental restoration, and daily necessities. In order to meet the large demand for microorganisms and microbial products, the cultivation of excellent microbial varieties is the key to microbial application. Microbial breeding methods are various, mainly physical mutagenesis, chemical mutagenesis and biological mutagenesis. Combined with the selection of microbial mutagenesis breeding methods in recent years, physical mutagenesis is most commonly seen. Microbial physical mutagenesis breeding refers to the use of physical factors to change its genetic characteristics, and then select individuals that meet the requirements of individuals from the mutant population, and then cultivate a breeding method for stable new varieties. Physical mutagenesis breeding is mainly used to transform microbial cell factories, such as the production of enzymes, amino acids, antibiotics, etc.; the production of biofuels such as butanol and ethanol; and the improvement of the characteristics of bacteria, drug resistance, and polarities; Petroleum degradation, fuel desulfurization, conversion and degradation of harmful substances in the soil, and microbial environmental restoration. Atmospheric pressure room temperature plasma (ARTP) mutagenesis technology has attracted more and more attention in the field of microbial mutation breeding and has become an important research technology. This paper mainly introduces the microbial physical mutagenesis technology, focuses on the application status of ARTP mutagenesis technology in microbial mutation breeding, and focuses on its improvement effect on spore-forming species, in order to achieve greater microbial mutation breeding.

\section{Principles and Methods of Common Physical Mutagenesis Technology}

Physical mutagenesis has the advantages of simple method, high safety, low cost and large harvest. Including UV mutagenesis, X-ray mutagenesis, $\gamma$-ray mutagenesis, fast neutron mutagenesis, space environment mutagenesis, ion beam injection mutagenesis, laser mutagenesis, microwave mutagenesis, ultra-high pressure mutagenesis, thermal effect mutagenesis, Mutagenesis techniques such as ionizing radiation mutagenesis, heavy ion radiation mutagenesis, ultrasonic wave array mutagenesis, and atmospheric pressure room temperature plasma (ARTP).

Physical factors such as ultraviolet rays, X-rays, gamma rays, fast neutrons and other particles, and microwave radiation can transmit energy to the living body through radiation, causing 
ionization and excitation of various molecules in the living body, resulting in a large number of chemically active freedoms. Atom or a radical. Among them, ultraviolet mutagenesis mainly causes DNA molecules to form pyrimidine dimers, weakens the hydrogen bond between double strands, causes distortion of double-stranded structures, and hinders normal pairing between bases; in addition, dimers also hinder double-stranded Unwinding, thus affecting DNA replication and transcription, causing mutation or death of the organism. Lasers can produce light, thermal and magnetic effects on cells. The space environment combines various physical factors such as radiation and microgravity to act on microbial cells.

ARTP relies on uniformly distributed high-concentration neutral active particles to alter microbial genetic traits, directly altering the molecular structure at the nucleotide level, such as causing DNA damage, causing transversion, transformation, transfer, insertion and deletion, or making circular plasmids Open loop, break or even break, or cause DNA double-strand or single-strand break. ARTP can also indirectly affect intracellular genetic material by acting on cells, and initiate various DNA repair mechanisms, such as SOS repair mechanisms, by using damage to whole cells. These inaccurate repairs trigger complex regulatory networks in biologically reactive cells. The genetic material and metabolic pathways are changed, so the mutant strain obtained by ARTP technology has good genetic stability. In addition, the atmospheric pressure room temperature of ARTP can change the permeability of plasma membrane, so that the cell matrix produces a large amount of active oxygen components into the cells, interacting with biological macromolecules, thereby changing the conformation and activity of macromolecules. People still need to know more about the mechanism of ARTP mutagenesis. Various studies have shown that the damage of DNA by ARTP technology is significantly higher than other physical and chemical mutagenesis methods.

\section{Application of ARTP Mutagenesis Technology}

The application of ARTP technology has greatly promoted the process of microbial mutation breeding. It is mainly reflected in improving the ability of microbial cell factories to produce microbial products, the improvement of microbial strains and the bioremediation of the environment.

The use of microorganisms can produce a variety of microbial products, such as biological enzymes, amino acids, antibiotics, microbial drugs, biofuels, food additives, and the like. ARTP technology can significantly improve the synthesis capabilities of microbial cell factories.

After the use of ARTP technology, the functional bioenzyme production performance of various microorganisms is greatly improved. Han Rui et al. used the Bacillus megaterium ATCC 14945 as the starting strain and combined with LiCl-UV-ARTP to obtain the mutant strain with the activity of penicillin $G$ acylase (PGA) 8.5 times higher than that of the original strain, which promoted the industrial production of PGA. . Fan Xinlei et al. performed ARTP mutagenesis on the Aspergillus niger strain, and obtained a mutant strain with an increase in glucose oxidase activity of $117.25 \%$ compared with the original strain. High-temperature protease is a product widely used in high-temperature washing, medicine, tanning, etc. Xue Gang et al. successfully used the ARTP technology to successfully breed high-yield mutants with a production activity of 1.56 times that of the original strain, which is a further development and application of high-temperature protease. Lay the foundation. Lactase plays a key role in the absorption of lactose by the human body. Qiu Wenwen and others used ARTP mutagenesis technology to screen yeast with high lactase activity, which greatly promoted the industrial production of lactase and optimization of dairy products. Nuclease P1 is essential for the production of food flavoring agents and fresheners. Liang Jianguang et al. performed ARTP mutagenesis on Penicillium citrinum strain CK-3 to obtain a mutant strain with a nuclease P1 enzyme activity increased by 42.2\%. Xue Zhenglian and others used ARTP mutagenesis technology to treat Bacillus licheniformis G2, and obtained a mutant strain with an esterase activity increased by $102 \%$ compared with the original strain. Cellulase is widely used in laundry, brewing and environmental management. UV-ARTP combined mutagenesis can be used to improve cellulase production strains, which can effectively improve the enzyme activity and yield 
of Penicillium oxalicum cellulase.

ARTP technology has a significant boost to a variety of biological amino acid synthesis capabilities. $\Gamma$-polyglutamic acid ( $\gamma$-PGA) has significant health care effects and has important application value. Shen Feixiang and others have induced $\gamma$-polyglutamic acid $(\gamma)$ by ammunizing Bacillus subtilis strain SY-ND by ARTP technology. -PGA) The $86.8 \%$ increase in the yield of the mutant strain reduced the production cost of $\gamma$-polyglutamic acid and its derivatives. Threonine is one of the essential amino acids in human body. It is widely used in food and medical fields. It can obtain fast metabolism, strong acid production, low $\mathrm{pH}$ resistance and good genetic stability through ARTP mutagenesis technology and high-throughput screening of 48-well plate. 1905\# mutant strain. Tryptophan can participate in the plasma protein renewal and synthesis of folic acid and heme in animals. It is widely used in medicine. Yang Lipeng and other breeding TRP-YP mutants with fast growth, stable genetics and high L-tryptophan production were selected by ARTP technology. -3-2. In addition, Zheng Mingying and others used the L-proline-producing strain as the starting strain for ARTP mutagenesis, and also obtained a mutant strain of Corynebacterium faecalis with a significantly improved L-valine yield.

Bacitracin is a kind of peptide antibiotic, which has a good inhibitory effect on Gram-positive bacteria, Gram-negative bacteria and actinomycetes, and often forms zinc bacitracin with zinc salt as a feed additive. Luo Xiuxiu et al. used ARTP mutagenesis technology to select a mutant strain with a $30 \%$ increase in the potency of the bacillus. Spinosad is a secondary metabolite produced by aerobic fermentation of S. spinosa, which has broad-spectrum high-efficiency, biodegradability and high safety. Qiao Changjun uses ribosome engineering theory combined with ARTP mutagenesis technology to screen out High-yield spinosad mutants contribute to the prevention and control of agricultural pests and diseases. ARTP-UV complex mutagenesis is also widely used in microbial mutation breeding. Shen Xiaojing et al. gave ARTP-UV compound mutagenesis treatment to erythromycin producing bacteria, and obtained a mutant strain of erythromycin producing bacteria with stable genetic stability and significantly improved yield.

ARTP technology has also had many successful examples in improving microbial drug synthesis. Oritavancin is a semi-synthetic glycopeptide antibiotic that acts as an antibacterial agent by inhibiting the biosynthesis of $\mathrm{G}+$ bacterial cell walls. Zheng Linghui and others used ARTP mutagenesis technology to treat Nocardia orientalis HS807-N-1287, and obtained high-yield mutant of Orivavans intermediate A82846B. Fusidic acid is highly sensitive to various Gram-positive bacteria related to skin infections, especially to Staphylococcus, and is often used as a dermatological drug. Zhang Jia et al. used ARTP-UV compound to induce the production of Fusidic acid. The mutant strain with a $14 \%$ increase in fermentation potency provides raw materials for the large-scale production of fusidic acid. Lovastatin can effectively lower blood cholesterol and low-density lipoprotein cholesterol levels, so it has a good preventive effect on atherosclerosis and coronary heart disease, so Putian sweet and so on with Monascus purpureus strain M-1 as the starting strain, ARTP mutagenesis was carried out to significantly increase Monacolin K production. Rapamycin (RAPA) is a novel macrolide immunosuppressant. RAPA blocks signaling by acting on cytokine receptors, which in turn blocks the progression of immune cells from G1 to S, resulting in immunosuppressive effects. Shi Yanman et al. combined the ARTP mutagenesis with UV mutagenesis to the mutagenic treatment of Actinobacillus actinomycetes BCLP-016, effectively screening out high-yield strains of rapamycin with five antibiotic resistances.

ARTP mutagenesis technology is also widely used in the production of oils, biofuels and food flavorings. The oil synthesis ability of Rhodotorula sinensis, Zhanjiang isochrysis, and Rhodospora erythraea was greatly improved after ARTP mutagenesis treatment. In terms of biofuel development, Wang Fengqin and others used ARTC-NTG complex mutagenesis as a starting strain of Bacillus typhimurium to obtain a mutant strain with a butanol yield increased by $21.6 \%-24.5 \%$. The ethanol production performance of alcohol yeast was also significantly better than that of the original strain by ARTP mutagenesis. It can be seen that the improvement of alcohol-producing bacteria by microbial mutation breeding can effectively alleviate the energy shortage problem and provide raw materials for the development of new energy sources. 
The long-term use of microbial strains and the damage of various breeding methods to the strains tend to reduce the production performance of the strains, and the strains are prone to death, fading, and variability. Therefore, the strains should be regularly tested in microbial production. The physiological indicators improve the production bacteria in time and optimize the culture conditions. ARTP mutagenesis can improve the tolerance, bacteriostatic and drug resistance of microbial strains to toxic substances, and help to cultivate excellent strains. In order to optimize the bio-production process of butanol, Zhang Jihua et al. used ARTP technology to mutagenize Clostridium beijerinckii, and screened mutant strains with good adaptability in medium with high phenol content, which improved the toxic substances of mutant strains. Tolerance. Dong Jing et al. used ARTP mutagenesis as a starting strain of Lactobacillus plantarum to significantly increase its growth rate and inhibition rate. Zhou Wenchen et al. performed ARTP mutagenesis on the biocontrol fungus T. harzianum to obtain a mutant strain with enhanced pesticide resistance and broad-spectrum antibacterial properties, which greatly improved the field adaptability of Trichoderma viride. In the optimization of straw mushroom species, He Jianhua used ARTP technology to construct a mutant strain of the excellent strain of straw mushroom, and obtained an excellent mutant strain which was resistant to low temperature and quick mushrooming.

With the rapid development of industry and agriculture and the improvement of human living standards, the pollution of the atmosphere, water, soil and food caused by various toxic substances such as waste gas, waste water, pesticides, and various kinds of garbage seriously affect people's lives and hinder social development. Microbial remediation refers to the process of removing pollutants from air, water and soil through the action of microorganisms, or making the pollutants harmless, and is used to alleviate and eradicate environmental pollution problems. Laccase can remove toxic substances such as lignin derivatives, tannins and phenolic compounds in wastewater, and plays an important role in improving food quality, paper industry, dye biological decolorization and wastewater treatment. Tian Jun et al. used the ARTP-UV complex mutagenesis method to mutagenize the alkaline-resistant laccase-producing fungus M5-3, and obtained a genetically stable mutant with an enzyme activity of $8.79 \mathrm{IU} / \mathrm{mL}$. The cellulase hydrolyzed lignocellulosic material can be used to produce bio-clean energy. The cellulase-producing strains were subjected to ultraviolet-lithium chloride-ARTP complex mutagenesis, and the enzyme activity was increased by 4.13\% compared with the original mutant strain. Mutant strain. Rhamnolipid has the function of repairing hydrocarbon pollutants, and the effect is good, and it is mostly used in oil exploitation. Wang Shuang et al. treated the Pseudomonas aeruginosa by UV-diethyl sulfate (DES)-ARTP compound mutagenesis, and obtained a mutant strain with a 5-fold increase in rhamnolipid production. Obviously, microbes have played an important role in environmental remediation, and applications will become more widespread.

\section{ARTP Superiority to Mutation Breeding of Bacillus}

Bacillus can produce spores, has the characteristics of heat and stress resistance, and has strong viability. It is widely used in the prevention of bioremediation and crop microbial diseases. The laboratory has long been engaged in the research of plant rhizosphere-promoting bacteria (PGPR), and screened out a large number of Bacillus strains with microbial bacterial fertilizer efficacy. The production of spores is conducive to the production, processing and survival, colonization and reproduction of fertilizers and fungi, with high production stability and good compatibility with chemical pesticides. Bacillus is easy to isolate and culture, can produce a variety of organic acids, inorganic acids, plant hormones and disease-resistant insecticides, so that soluble phosphates and silicates dissolve, promote crop growth, effectively reduce the use of chemical fertilizers and pesticides, with good Prospects. Therefore, it is essential to optimize the transformation of Bacillus. ARTP has many advantages in microbial breeding. If the operation is simple, it has no harm to the human body, no pollution to the environment; the mutagenic temperature is low, it is carried out under normal pressure, and the active particle composition is diverse and the concentration is high; the biological damage mechanism is diverse, and the mutation diversity is especially suitable for the metabolic network complex. Microorganisms. ARTP technology features are suitable for improved 
breeding of spore-forming bacteria. Han Rui et al. used ARTP technology to transform Bacillus megaterium to obtain a high-yield mutant of penicillin $G$ acylase. Xue Zhenglian and others used ARTP technology to transform Bacillus licheniformis to obtain mutant strains with high esterase activity. Similarly, Shen Feixiang and others performed ARTP mutagenesis on Bacillus subtilis, and also obtained a high-yield mutant of $\gamma$-polyglutamic acid. It can be seen that the development of ARTP mutation breeding technology provides a convenient and efficient breeding method for Bacillus breeding.

\section{Conclusion}

The development of microbial mutation breeding methods tends to be simple, safe, efficient, oriented and environmentally friendly. From the original natural selection to artificial breeding, mutagenesis and selection; from single mutagenesis to compound mutagenesis; from irrational mutagenesis to targeted mutagenesis. Microbial mutation breeding technology has been perfected. In the selection of mutagenesis methods, according to the needs of experiments, a variety of mutagenesis methods are often combined to screen out mutant strains that meet the requirements of people. At present, mutagenesis breeding is mostly used for the transformation of microbial cell factories and microbial drug production strains, but has less application in bioremediation, biofuels and waste degradation, especially in the breeding of important functions of Bacillus, future ARTP technology. There is also a larger application space in these directions. In addition to the application of ARTP technology in microbial mutation breeding, it should also broaden its application fields, try to integrate animal and plant cells, molecular transformation at the nucleic acid and protein levels, directed induction of embryonic stem cells in medicine, and synthetic development of new biological materials. The application of ARTP technology to maximize the benefits of its application. At present, ARTP technology has many advantages and has achieved a lot of results. With the further improvement, the mutagenic efficiency will be higher and higher, and the application range will be wider and wider.

\section{References}

[1] Wang Xingli, Li Zongwei, Chen Linhai, et al. New Progress in Industrial Microbial Physical Mutation Breeding Technology [J]. Biotechnology Bulletin, 2007, (2): 114-118.

[2] Liu L, Liu Y, Shin HD, et al. Developing Bacillus spp. as acell factory for production of microbial enzymes and industrially important biochemicals in the context of systems and synthetic biology [J]. Applied Microbiology \& Biotechnology, 2013, 97(14): 6113-6127.

[3] Fontus M, Ortoleva P. Electrophysiological-metabolic modeling of microbes: applications in fuel cells and environmentanalysis [J]. Methods in Molecular Biology, 2012, 881: 411-431.

[4] Zhang Xue, Zhang Xiaofei, Wang Liyan, et al. Advances in biological mutation breeding and its application in atmospheric pressure room temperature plasma[J].Journal of Chemical Industry and Engineering(China),2014,(7):2676-2684. 